Noel W.M. Shek*, Tiong Y. Tan, Simon C.K. Ding, Brian H.Y. Chung, Elizabeth T.K. Lau and Mary H.Y. Tang

\title{
Prenatal diagnosis of agenesis of the corpus callosum and cerebellar vermian hypoplasia associated with a microdeletion on chromosome
}

\section{$1 \mathrm{p} 32^{\mathrm{a}}$}

\begin{abstract}
We present the prenatal detection of a 1p32.1p31.3 microdeletion $(3.46 \mathrm{Mb})$ by array comparative genomic hybridization (CGH) associated with fetal agenesis of the corpus callosum (ACC) and cerebellar vermian hypoplasia. Analysis of cultured amniocytes showed a normal karyotype. Our observations strengthen the association between this locus and central nervous system development. In addition, the fetus reported herein underscores the importance of array CGH analysis when ACC is detected prenatally, especially when there are additional central nervous system anomalies, to search for submicroscopic imbalances which can facilitate further management and parental counselling. Moreover, the presence of urinary tract anomalies should alert the clinician to the possibility of a $1 p$ interstitial deletion, although the absence of such does not exclude it. Further reports will help to provide more information on the long-term outcomes of individuals with such microdeletion as there are only limited data.
\end{abstract}

Keywords: Agenesis of the corpus callosum; array CGH; microdeletion; prenatal diagnosis.

\footnotetext{
a Informed consent was obtained from the patient for publication of this case report and accompanying images.

*Corresponding author: Dr. Noel W.M. Shek, Department of Obstetrics and Gynaecology, Queen Mary Hospital, The University of Hong Kong, 102 Pokfulam Road, Hong Kong SAR, China, Tel.: +852 22553914, Fax: +852 25173278, E-mail: sheknoel@hku.hk Tiong Y. Tan: Department of Paediatrics and Adolescent Medicine, Queen Mary Hospital, The University of Hong Kong SAR, China; Department of Biochemistry, The University of Hong Kong, Hong Kong SAR, China; and Genetic Health Services Victoria, Murdoch Children's Research Institute, Royal Children's Hospital, Melbourne, Australia Simon C.K. Ding: Prenatal Diagnostic and Counselling Department, Tsan Yuk Hospital, The University of Hong Kong, Hong Kong, SAR, China Brian H.Y. Chung: Department of Paediatrics and Adolescent Medicine, Queen Mary Hospital, The University of Hong Kong SAR, China Elizabeth T.K. Lau and Mary H.Y. Tang: Department of Obstetrics and Gynaecology, Queen Mary Hospital, The University of Hong Kong SAR, China; and Prenatal Diagnostic and Counselling Department, Tsan Yuk Hospital, The University of Hong Kong, Hong Kong, SAR, China
}

\section{Introduction}

We present the prenatal detection of a $1 \mathrm{p} 32.1 \mathrm{p} 31.3$ microdeletion by array comparative genomic hybridization (CGH) associated with fetal agenesis of the corpus callosum and cerebellar vermian hypoplasia. We also review the literature. Our observations strengthen the association between this locus and central nervous system (CNS) development, and suggest that investigation for submicroscopic chromosomal aberrations is recommended for such fetuses to facilitate further management and parental counselling.

\section{Case presentation}

A 37-year-old, gravida 3, para 0, woman was seen at 18 weeks of gestation for increased risk on combined trisomy 21 screening (1 in 180). Her two previous pregnancies were terminated in the first trimester for social reasons. Aside from borderline hypertension, she was otherwise healthy. Gestational diabetes diagnosed in the second trimester was managed with dietary control. Analysis of cultured amniocytes obtained by amniocentesis showed a normal karyotype 46,XX. The morphology scan at 18 weeks of gestation was limited by the prone position of the fetus; therefore ultrasound scan was repeated at 22 weeks. The ultrasound scan demonstrated prominent cerebral lateral ventricles and agenesis of the corpus callosum (ACC), including absent cavum septum pellucidum, "tear-drop" sign (colpocephaly), high-riding third ventricle, "steerhorn" configuration of frontal horns and absent pericallosal artery (Figure 1A-C). Partial absence of the cerebellar vermis was suspected (Figure 1D). In addition, fetal biometry was 1-2 weeks behind. Fetal magnetic resonance imaging (MRI) performed at 23 weeks demonstrated the absence of the cerebellar vermis without ballooning of 


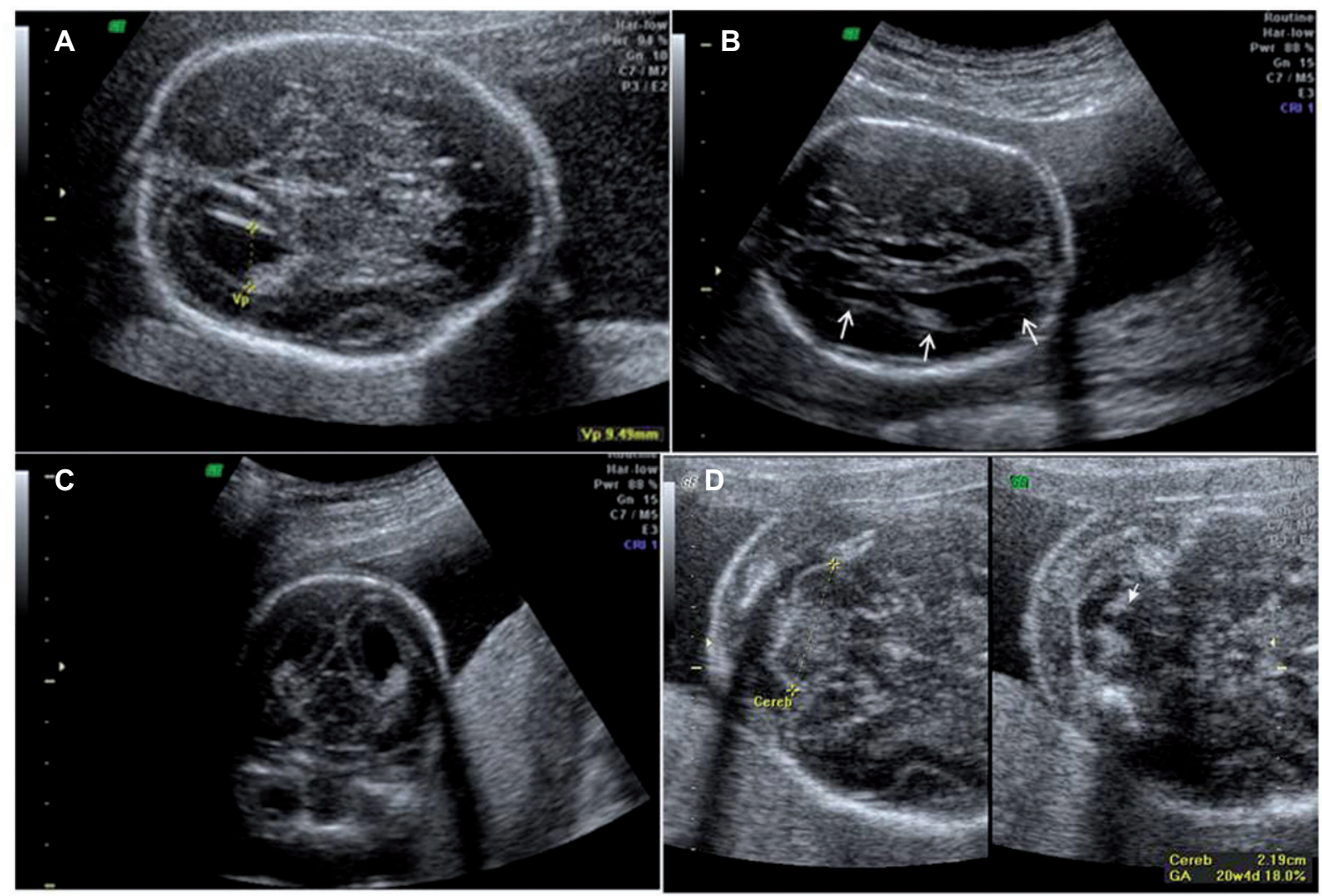

Figure 1 (A-D) Ultrasound pictures of fetal brain at 22 weeks. (A) Prominent cerebral lateral ventricle. (B) Colpocephaly, "tear-drop" sign. (C) "Steerhorn" configuration of frontal horns. (D) Partially absent cerebellar vermis.

the posterior fossa, and complete ACC (Figure 2A-E). The couple opted to terminate the pregnancy by misoprostol induction at 23 weeks 4 days. Postmortem examination of the abortus confirmed the absence of the cerebellar vermis and the corpus callosum. No dysmorphic features or other abnormalities were detected.

DNA was extracted from amniotic fluid cells using the Gentra Puregene Kit (Qiagen, Germantown, MD, USA) and subjected to array CGH study. Array CGH was performed using the NimbleGen CGX-135K array (Roche Diagnostics $\mathrm{GmbH}$, Madison, WI, USA) with maximum probe spacing at one every $10 \mathrm{~kb}$ in the clinical regions and one every $35 \mathrm{~kb}$ throughout the rest of the genome. Test DNA and reference female DNA (Promega, Madison, WI, USA) were labeled with $\mathrm{Cy} 3$ and $\mathrm{Cy} 5$, respectively, and hybridized according to the manufacturer's instructions. Array data were analyzed by the Genoglyphix software (Signature Genomics, Spokane, WA, USA). A de novo 3.46-Mb interstitial deletion of chromosome 1p32.1-1p31.3 was detected (chr1: 60638478-64100969, chromosome build hg18 Mar. 2006; Figure 3A). One hundred and sixty four probes were involved in the deletion region which includes, amongst 15 other genes, the nuclear factor IA (NFIA) gene. Deletion was confirmed by fluorescent in situ hybridization (FISH) using probe from BAC clone RP11-31P4 (The Hospital for Sick Children, Toronto, Canada; Figure 3B).

\section{Discussion}

The corpus callosum is an important commissural structure connecting the two cerebral hemispheres and is involved in integrating motor, sensory and cognitive function. In human embryos, it begins to develop from 11 to 12 weeks' gestation and is completely formed by 20 weeks' gestation. ACC occurs with an incidence of 1 per 4000 livebirths and a prevalence as high as 3-5\% in individuals with neurodevelopmental disabilities [3]. ACC can be associated with other cerebral and extracerebral malformations. In addition, ACC can be associated with chromosomal rearrangements [7] and can occur as a component of genetic syndromes [6] and metabolic conditions [1]. Its causal heterogeneity and variable phenotypic expression have limited the identification of causative genes. Twelve distinct genomic loci have been identified to be associated 

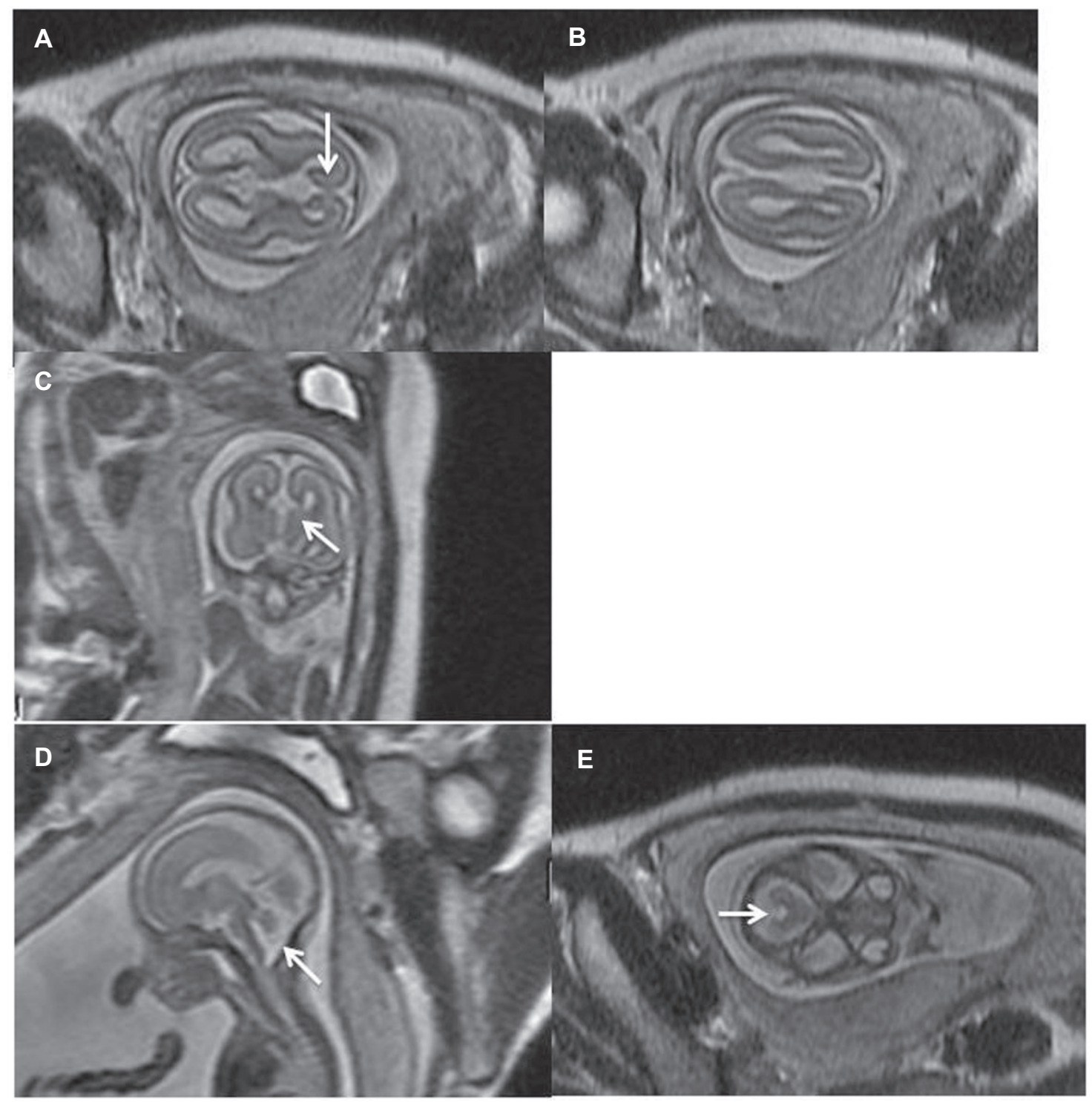

Figure 2 (A-E) Magnetic resonance imaging pictures of fetal brain at 23 weeks. (A, B) Parallel cerebral lateral ventricles and high-riding third ventricles (axial images). (C) Absent corpus callosum and high-riding third ventricle (coronal image). (D) Absent cerebellar vermis (sagittal image). (E) Absent cerebellar vermis (axial image).

consistently with ACC, and at least 30 other recurrent loci which may contain genes that contribute to ACC have been identified [10]. Furthermore, many ACC loci confer susceptibility to other brain anomalies, such as cerebellar hypoplasia, microcephaly and polymicrogyria [10]. Recently, a novel 4.93-Mb 1p31.3p32.2 deletion involving the NFIA gene was reported by Koehler et al. [8] in association with macrocephaly and hypoplasia of the corpus callosum. Mild cerebellar vermian hypoplasia was also described in their postnatally ascertained patient.

Interstitial deletions of different sizes involving $1 \mathrm{p} 31$ and $1 \mathrm{p} 32$ in the short arm of chromosome 1 are rare and have been described in six reports involving seven patients
$[2,4,5,8,9,11]$ including the one described by Koehler et al. [8] and a fetus recently reported by Chen et al. [5]. Individuals with haploinsufficiency of the NFIA gene shared corpus callosal abnormalities and partly shared other CNS malformations including ventriculomegaly, congenital hydrocephalus, Chiari type I malformation and tethered spinal cord. Developmental delay was observed in all of these patients. Three of the six also had urinary tract defects, including vesicoureteric reflux (VUR) [4, 9]. The NFIA gene at 1 p31.3 encodes a member of the nuclear factor I family of transcription factors. Disruption or deletion of the NFIA gene is associated with a syndrome including brain anomalies (abnormal corpus callosum, hydrocephalus, 
A
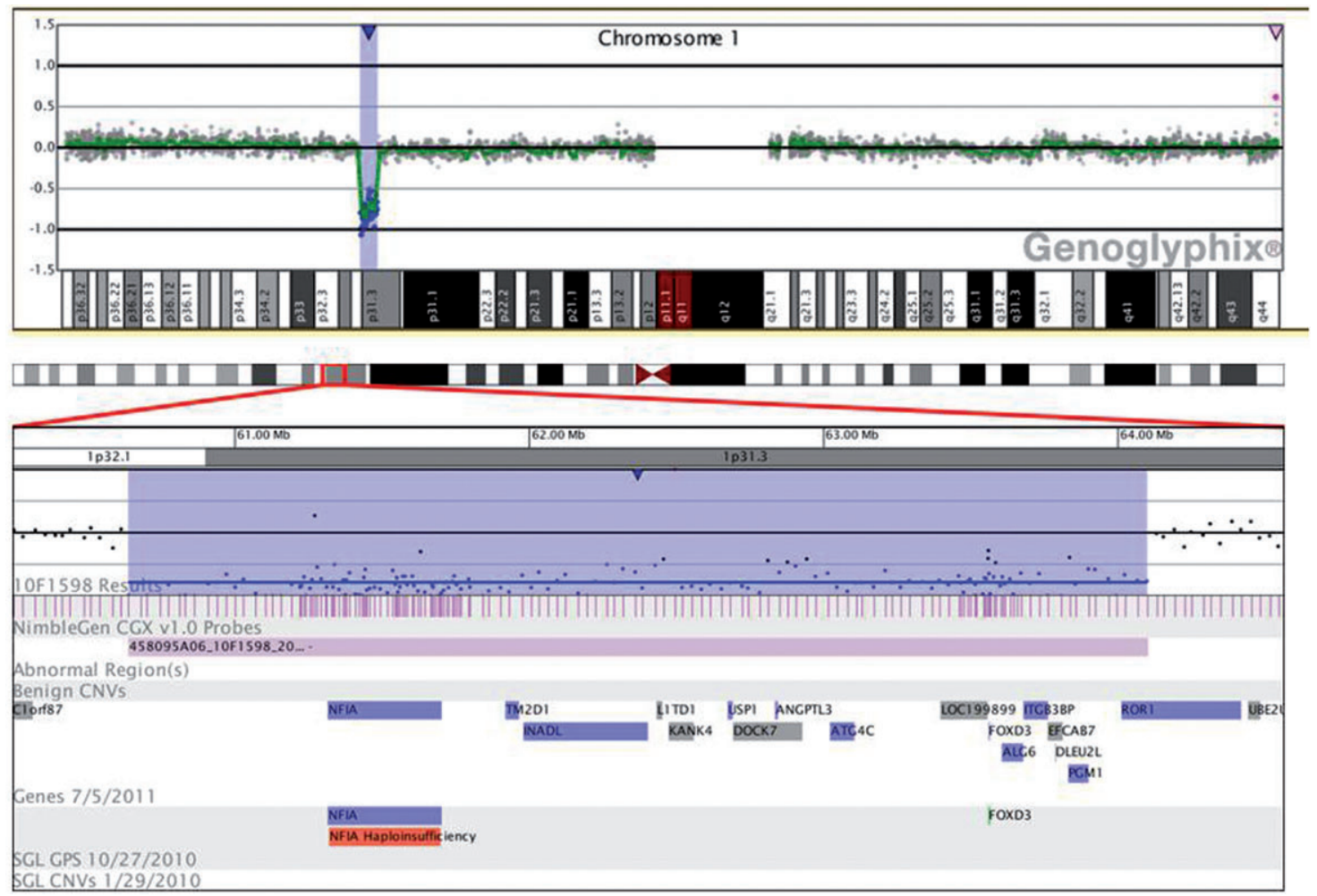

1p32.1 -1p31.3 deletion

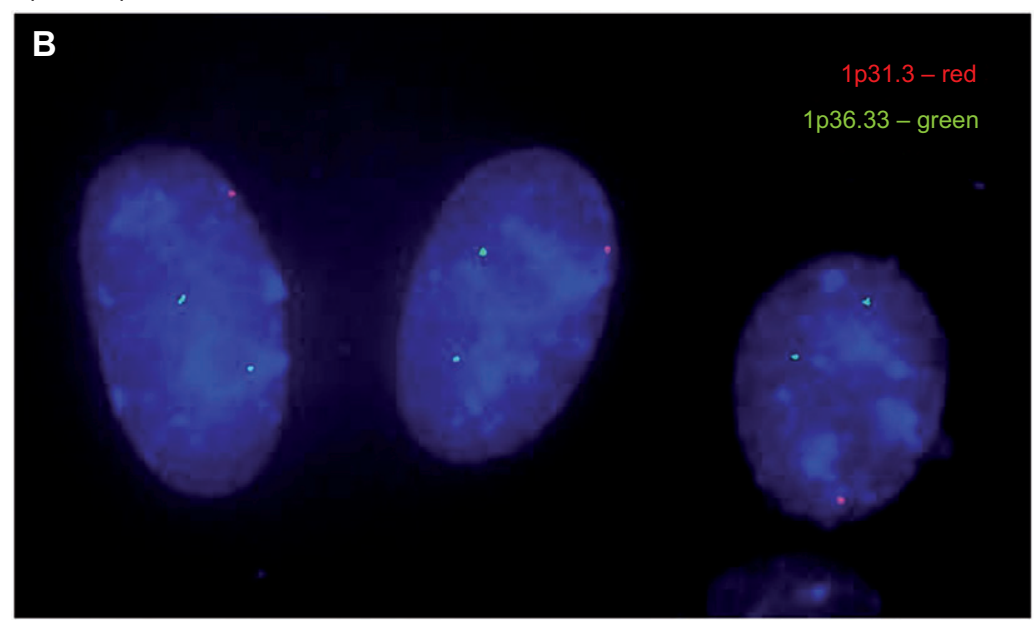

Figure 3 Results from array CGH and FISH showing deletion at chromosome 1p32.1-1p31.3. (A) Each dot on the $X$-axis represents one oligonucleotide probe on the respective chromosome position. Copy loss at 1p32.1-1p31.3 can be visualized in the lower panel covered by 146 probes and 16 genes including NFIA. (B) FISH using probe RP11-31P4 (red) at 1p31.3 confirms the loss of a 1p31.3 signal; control probe RP11-465B22 (green) located 2 signals for 1 p36.33.

ventriculomegaly and Chiari type I malformation), spinal abnormalities (tethered spinal cord) and urinary tract abnormalities (VUR, cystic kidney disease, renal agenesis and insufficiency) [9]. The knockout mice model of NFIA deficiency also has absence of the corpus callosum and CNS anomalies, with urinary tract defects [9].
Our report of ACC and cerebellar vermian hypoplasia in association with a 3.46-Mb $1 \mathrm{p} 32.1 \mathrm{p} 31.3$ deletion represents one of the early reports of prenatal detection of this deletion by array CGH analysis. The fetus reported here has the smallest deletion reported prenatally so far. Additional CNS malformations typically described 


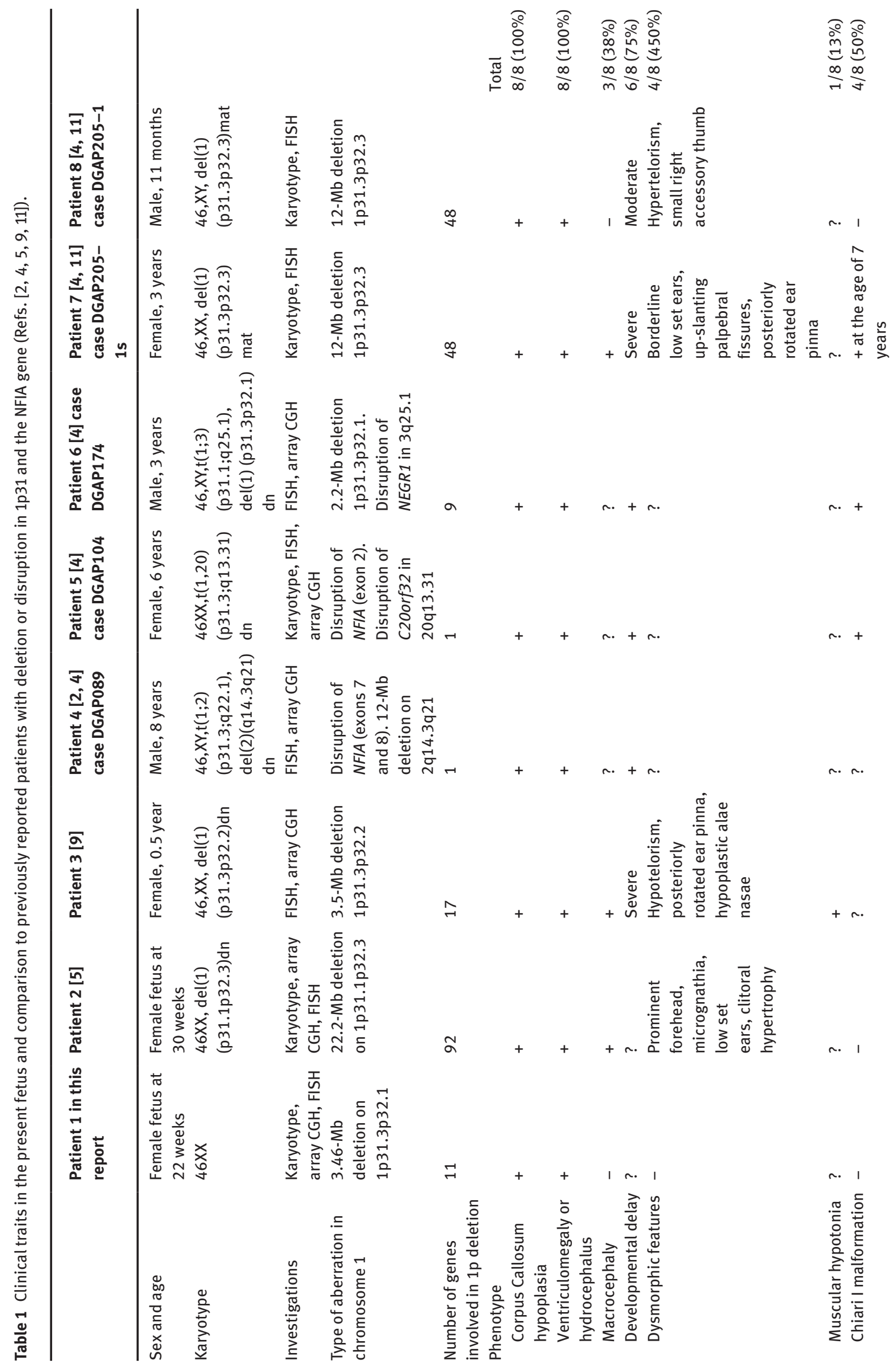


in association with similar deletions were absent in the fetus reported here, and no urinary tract abnormalities were detected during sonography or postmortem examination. It is possible that the presence of urinary tract anomalies detected in other reported patients could be due to the involvement of other genes in the deletion interval. However, as stated by Lu et al. [9], subtle anatomical defects in the ureter or kidney are often subclinical and may exist below the limit of detection. This may be true for fetuses in the second trimester. Table 1 summarizes the clinical traits of present fetus and reported patients.

\section{Conclusion}

In the presence of ACC on prenatal ultrasonography, a diligent search for other CNS anomalies and extracerebral malformations is warranted. Fetal MRI helps in confirming the diagnosis and in identifying associated brain anomalies. The fetus reported herein underscores the importance of array CGH analysis when ACC is detected prenatally, especially when there are additional CNS anomalies or growth restriction, to search for submicroscopic imbalances. The presence of urinary tract anomalies should alert the clinician to the possibility of a $1 p$ interstitial deletion, but the absence of such does not exclude it. Prenatal detection of submicroscopic copy number abnormalities can facilitate informed decisionmaking by parents. However, information on long-term outcomes in rare syndromes such as the 1 p32 deletion is limited, and most data are from postnatally ascertained children. Further reports will help to clarify the situation.

Acknowledgments: We thank Dr Tina Lam of the Department of Radiology, Queen Mary Hospital, for providing the MRI images.

\section{Conflict of interest statement}

Authors' conflict of interest disclosure: The authors have stated explicitly that there are no conflicts of interest in connection with this article.

Research funding: The authors have declared that there was no special source of funding supporting this work or publication.

Received May 10, 2012. Accepted January 4, 2013. Previously published online March 14, 2013. 


\section{References}

[1] Bamforth F, Bamforth S, Poskitt K, Applegarth D, Hall J. Abnormalities of corpus callosum in patients with inherited metabolic disease. Lancet. 1988;2:451.

[2] Barton JS, O'Loughlin J, Howell RT, L'e Orme R. Developmental delay and dysmorphic features associated with a previously undescribed deletion on chromosome 1. J Med Genet. 1995;32:636-7.

[3] Bodensteiner J, Schaefer GB, Breeding L, Cowan L. Hypoplasia of the corpus callosum: a study of 445 consecutive MRI scans. J Child Neurol. 1994;9:47-9.

[4] Campbell CGN, Wang H, Hunter GW. Interstitial microdeletion of chromosome 1p in two siblings. Am J Med Genet. 2002;111:28994.

[5] Chen CP, Su YN, Chen YY, Chern SR, Liu YP, Wu PC, et al. Chromosome 1p32-p31 deletion syndrome: prenatal diagnosis by array comparative genomic hybridization using uncultured amniocytes and association with NFIA haploinsufficiency, ventriculomegaly, corpus callosum hypogenesis, abnormal external genitalia, and intrauterine growth restriction. Taiwan J Obstet Gyne. 2011;50:345-52.

[6] Cohen MM Jr., Kreiborg S. Agenesis of the corpus callosum. Its associated anomalies and syndromes with special reference to Apert syndrome. Neurosurg Clin N Am 1991;2: 565-8.

[7] Dobyns WB. Absence makes the search grow longer. Am J Hum Genet. 1996;58:7-16.

[8] Koehler U, Holinski-Feder E, Ertl-Wagner B, Kunz J, von Moers A, von Voss $\mathrm{H}$, et al. A novel 1p31.3p32.2 deletion involving NFIA gene detected by array CGH in a patient with macrocephaly and hypoplasia of the corpus callosum. Eur J Paediatr. 2010;169:463-8.

[9] Lu W, Quintero-Rivera F, Fan Y, Alkuraya FS, Donovan DJ, Xi $Q$, et al. NFIA haploinsufficiency is associated with a CNS malformation syndrome and urinary tract defects. PLoS Genet. 2007;3:e80.

[10] O'Driscoll MC, Black GCM, Clayton-Smith J, Sherr EH, Dobyns WB. Identification of genomic loci contributing to agenesis of the corpus callosum. Am J Med Genet. 2010;152A:2145-59.

[11] Shanske AL, Edelmann L, Kardon NB, Grosset P, Levy B. Detection of an interstitial deletion of 2q21-22 by high resolution comparative genomic hybridization in a child with multiple congenital anomalies and an apparent balanced translocation. Am J Med Genet. 2004;131A:29-35. 